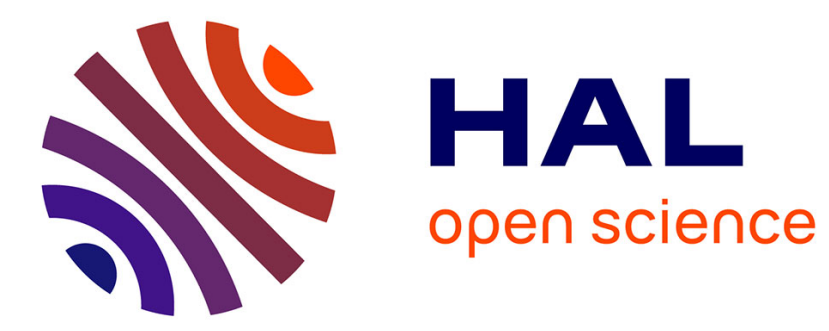

\title{
Sparse Deconvolution for Moving-Source Localization
}

Mai Quyen Pham, Benoit Oudompheng, Barbara Nicolas, Jerome I. Mars

\section{To cite this version:}

Mai Quyen Pham, Benoit Oudompheng, Barbara Nicolas, Jerome I. Mars. Sparse Deconvolution for Moving-Source Localization. ICASSP 2016 - 41st IEEE International Conference on Acoustics, Speech and Signal Processing, Mar 2016, Shanghai, China. pp.355-359, 10.1109/ICASSP.2016.7471696 . hal01343760

\section{HAL Id: hal-01343760 \\ https://hal.science/hal-01343760}

Submitted on 26 Jul 2016

HAL is a multi-disciplinary open access archive for the deposit and dissemination of scientific research documents, whether they are published or not. The documents may come from teaching and research institutions in France or abroad, or from public or private research centers.
L'archive ouverte pluridisciplinaire $\mathbf{H A L}$, est destinée au dépôt et à la diffusion de documents scientifiques de niveau recherche, publiés ou non, émanant des établissements d'enseignement et de recherche français ou étrangers, des laboratoires publics ou privés. 


\title{
SPARSE DECONVOLUTION FOR MOVING-SOURCE LOCALIZATION
}

\author{
Mai Quyen Pham ${ }^{1}$, Benoit Oudompheng ${ }^{1,2}$, Barbara Nicolas ${ }^{3}$, and Jérôme I. Mars ${ }^{1}$
}

\author{
${ }^{1}$ Univ. Grenoble Alpes, GIPSA-Lab \\ 11 Rue des Mathématiques, \\ 38402 Saint-Martin d'Héres, France \\ $\{$ mai-quyen.pham, \\ jerome.mars\}@gipsa-lab.grenoble-inp.fr
}

\author{
${ }^{2}$ MicrodB \\ 28 Chemin du Petit Bois, \\ 69134, Écully, France \\ benoit.oudompheng@gipsa-lab.grenoble- \\ inp.fr
}

\author{
3 \\ Université de Lyon \\ CREATIS, CNRS UMR5220 \\ 7 Avenue Jean Capelle, \\ 69621 Villeurbanne Cedex, France \\ barbara.nicolas@creatis.insa-lyon.fr
}

\begin{abstract}
In this paper, we propose a method for moving-source localization based on beamforming output and on sparse representation of the source positions. The goal of this method is to achieve spatial deconvolution of the beamforming, to provide accurate source localization for pass-by experiments. To perform this deconvolution, we use a smooth approximation of $\ell_{1} / \ell_{2}$ [1], which is well suited for the recovery of sparse signals. We validate this method on simulated data, and compare it to the DAMAS-MS method [2], one of the classical methods used in beamforming deconvolution.
\end{abstract}

Index Terms - Smoothed $\ell_{1} / \ell_{2}$ regularization, Sparse representation, Moving-source localization, Beamforming deconvolution, Acoustic signal processing

\section{INTRODUCTION}

Source localization is a classical problem in underwater acoustics. Many methods have been developed to solve this based on array processing. The most classical is beamforming [3] which consists in summing delayed signals. This method has been extensively used due to its robustness against noise and environmental mimatch. For pass-by experiments, due to the moving of the acoustic sources, classical beamforming cannot be used, and source mapping is achieved by beamforming for moving sources (BF-MS) [2].

Nevertheless, BF-MS spatial resolution is limited, as the image of a point source is the array transfer function, which is comprised of a main lobe and secondary lobes. Consequently, improvements have been proposed to overcome this problem, among which there are deconvolution methods.

For moving source, Sijtsma recently proposed an extended version of the deconvolution method CLEAN [4], known as CLEAN-SC [5], with an approach similar to the Matching Pursuit [17]. These methods provides satisfactory results for high signal-to-noise ratios (SNRs). Fleury and Bulté developped another approach DAMAS-MS [2], an extended version of DAMAS [6, 7] which can improve moving-source localization in a high SNR context.

In the case of low SNR, the problem is difficult to solve,
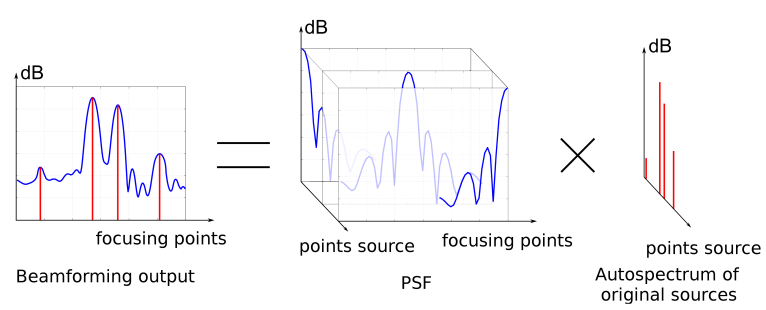

Fig. 1. Modelization of the forward problem

and some other approaches need to be developed. For example, a regularization term can be included to stabilize the solution. Basically, the Tikhonov regularization is applied to jet noisesource localization [8]. The sparse distribution of sources is also commonly used [9, 10, 11, 12, 13], although these methods have been developed for fixed-source localization.

The goal of the present paper is to propose a new deconvolution method 1 to apply to BF-MS results to improve moving-source localization.

The strategy is to formulate the forward problem as an optimization problem, with constraints derived from the physical context. The proposed cost function contains several parts: (i) a data-fidelity term accounts for the noise characteristics; (ii) the smoothed $\ell_{1} / \ell_{2}$ ratio [1] promotes sparse representation of moving-source locations; and (iii) the knowledge of some physical properties of sources is also considered, such as constraints on its maximal and minimal amplitudes.

The paper is organized as follows: section 2 presents the proposed forward model; section 3 describes the minimization problem, the proposed algorithm, and some mathematical tools essential to this methodology; and simulation results are evaluated both objectively and subjectively in Section 4, and compared to the DAMAS-MS performance.

\section{OBSERVATION MODEL}

BF-MS compensates the Doppler effect and back-propagates the pressures measured by the $M$ sensor array to a calculation

\footnotetext{
${ }^{1}$ This work was financially supported by the Ministère du Redressement Productif and by the DGA-MRIS, grant RAPID ARMADA.
} 
grid of $N$ points, which correspond to the possible source locations. We consider the classical case in pass-by experiments of sources, in the far-field, sharing the same movement and with low Mach numbers, $\|\overrightarrow{M a}\| \ll 1$. For these conditions, some assumptions can be made during short time intervals of duration $T$, which are referred to as snapshots [2]:

1) The sources are in fixed positions;

2) The Doppler effect is negligible at the frequencies and speeds of interest (i.e., it does not exceed the frequency resolution defined for the localization results).

Under these assumptions, BF-MS can be implemented in a simple way in the frequency domain. The measured acoustic pressures are temporally sliced into $K$ snapshots that are indexed by $k$. The calculation grid of $N$ points is defined for the snapshot $k$. Note that this grid moves according to the known global movement of the sources.

The beamforming computed for the $n^{\text {th }}$ calculation point at the frequency $\zeta$ and for the snapshot $k$ is given by:

$$
b_{n}^{k}(\zeta)=\left(\mathbf{w}_{n}^{k}\right)^{*} \mathbf{p}^{k}(\zeta)\left(\mathbf{p}^{k}(\zeta)\right)^{*} \mathbf{w}_{n}^{k}
$$

where $\cdot^{*}$ is the conjugate transpose, $\mathbf{p}^{k}(\zeta)$ is the $M \times 1$ vector of the Fourier transforms of the pressures measured by the sensors during the snapshot $k$ at frequency $\zeta$, and $\mathbf{w}_{n}^{k}$ is the steering vector of size $M \times 1$ between the $M$ sensors and the $n^{\text {th }}$ calculation point. The $m^{\text {th }}$ element $w_{n m}^{k}$ of $\mathbf{w}_{n}^{k}$ is:

$$
w_{n m}^{k}=\left(\sum_{m^{\prime}=1}^{M}\left(\frac{1}{R_{n m^{\prime}}^{k}}\right)^{2}\right)^{-1} \frac{\exp \left(-j \zeta R_{n m}^{k}\right)}{R_{n m}^{k}}
$$

where $j$ is a complex number, and $R_{n m}^{k}$ is the distance between the $m^{\text {th }}$ sensor and the $n^{\text {th }}$ calculation point during the snapshot $k$. We then define the vector of size $\mathbf{b}(\zeta) \in \mathbb{R}^{N}$ where its $n^{\text {th }}$ element $b_{n}(\zeta)$ is the estimate of the BF-MS output for the $n^{\text {th }}$ calculation point, through averaging over all the $K$ snapshots:

$$
b_{n}(\zeta)=\frac{1}{K} \sum_{k=1}^{K} b_{n}^{k}(\zeta)
$$

Note that in the case considered, the sensor array is a linear array along the $x$-axis. Consequently, BF-MS is performed along the $x$ dimension, and the calculation grid is a one-dimension vector of size $N \mathrm{x} 1$ along $x$.

We set the following assumptions:

(H1): The sources are random variables that are mutually independent and stationary;

(H2): The number $M$ of sensors is greater than the number $N_{s}$ of sources $\left(M>N_{s}\right)$, and these $N_{s}$ sources are sparsely distributed on the calculation grid;

(H3): The noise components are Gaussian with zero mean, mutually independent, and independent of the sources.

On this basis, we can model the beamforming output at a given frequency $\zeta$ :

$$
\mathbf{b}(\zeta)=\mathbf{H}(\zeta) \overline{\mathbf{a}}(\zeta)+\mathbf{e}(\zeta)
$$

where $\mathbf{H}(\zeta) \in \mathbb{R}^{N \times N}$ (Fig. 11-middle) is the array transfer function matrix that contains the beamforming point-spread functions. The $\left(n, n^{\prime}\right) \in\{1, \ldots, N\}^{2}$ element of $\mathbf{H}(\zeta)$ is:

$$
h_{n n^{\prime}}(\zeta)=\frac{1}{K} \sum_{k=1}^{K}\left|\sum_{m=1}^{M}\left(w_{n m}^{k}\right)^{*} \frac{\exp \left(-j \zeta R_{n^{\prime} m}^{k}\right)}{R_{n^{\prime} m}^{k}}\right|^{2}
$$

and $\mathbf{e}(\zeta) \in \mathbb{R}^{N}$ is the autospectra of the measurement noise, and $\overline{\mathbf{a}}(\zeta) \in \mathbb{R}^{N}$ is the autospectra of the possible sources located at the $N$ calculation points (Fig. 1 right), which we want to recover. More details on the forward model are given in [2, 14].

\section{PROPOSED METHOD}

\subsection{Criterion to be minimized}

In the following, we omit the frequency $\zeta$ for the sake of simplicity. The purpose of this study is to identify $\overline{\mathbf{a}}$ from b through Equation (4), which leads to an inverse problem. To solve this, we propose an optimization objective that minimizes the following criterion:

$$
\underset{\mathbf{a} \in \mathbb{R}^{N}}{\operatorname{minimize}} \frac{1}{2}\|\mathbf{H a}-\mathbf{b}\|^{2}+\lambda \varphi(\mathbf{a})+\rho(\mathbf{a})
$$

The term $\varphi$ models a regularization function that accounts for the sparsity of the solution with the regularization parameter $\lambda \in[0,+\infty[$. When $\lambda=0$, no regularization issue is considered, as presented in [2]. If $\varphi=\ell_{2}^{2}$, the solution can be achieved using an adapted Tikhonov regularization method. This regularization function is not adapted for the considered case of sparse source positions. Another possibility is to choose $\varphi=\ell_{1}$-norm to restore the sparsity of solution, as was proposed in [9, 13]. In the present paper, we propose to use a new regularization, the smoothed $\ell_{1} / \ell_{2}$ ratio proposed in [1]. To validate the choice of this regularization term for the case of sparse localization of acoustic moving sources, we investigate two possibilities for $\varphi$ : For every $\mathbf{a} \in \mathbb{R}^{N}$ :

1 . The smooth approximation of $\ell_{1}$-norm is named $\ell_{1, \alpha}$, where $\ell_{1, \alpha}(\mathbf{a})=\sum_{n=1}^{N}\left(\sqrt{a_{n}^{2}+\alpha^{2}}-\alpha\right)$, is one classical choice for sparse representation.

2. The smoothed $\ell_{1} / \ell_{2}$ ratio as proposed in [1]: $\log \left(\frac{\ell_{1, \alpha}(\mathbf{a})+\beta}{\ell_{2, \eta}(\mathbf{a})}\right)$ with, $\left.\ell_{2, \eta}(\mathbf{a})=\sqrt{\sum_{n=1}^{N} a_{n}^{2}+\eta^{2}},(\alpha, \beta, \eta) \in\right] 0,+\infty\left[^{3}\right.$. $\left.\left.\rho: \mathbb{R}^{N} \rightarrow\right]-\infty,+\infty\right]$ introduces the prior knowledge on the sources. We assume that $\rho$ is a proper, lower semicountinuous, convex function, continuous in its domain $(\operatorname{dom} \rho)$. One natural choice for $\rho$ is the indicator function of the hypercube $\left[a_{\min }, a_{\max }\right]^{N}$ (equal to 0 if $\forall n \in\{1, \ldots, N\}, a_{n} \in\left[a_{\min }, a_{\max }\right]$, and $+\infty$ otherwise), where $a_{\min }$ (resp. $a_{\max }$ ) is the lower (resp. upper) boundary of $\overline{\mathbf{a}}$. In practice, we choose $a_{\min }$ as 0 , which leads to a nonnegative constraint on the source power variables, and $a_{\max }$ is the maximum value of $\mathbf{b}$.

In the following, we denote:

$$
\psi(\mathbf{a})=\frac{1}{2}\|\mathbf{H a}-\mathbf{b}\|^{2}+\lambda \varphi(\mathbf{a}) .
$$




\subsection{Variable metric forward-backward algorithm}

To provide a numerical solution for Equation (6), it is necessary to minimize a nonconvex and nonsmooth criterion. If the function $\rho$ in (6) only imposes the non negativity of the solution, the Multiplicative Iterative (MI) algorithm [18] can be used. However, when more information on the solution is available, e.g. the upper boundary of the solution, this algorithm is no more appropriate. We then use the variable metric forward-backward (VMF-B) algorithm proposed in [15] to take into account all the available information on the solution. This algorithm requires two optimization principles. The first is related to the choice of a variable metric that relies upon majorization-minimization properties; i.e.:

Definition 1 Let $\psi: \mathbb{R}^{N} \rightarrow \mathbb{R}$ be a differentiable function. Let $\mathbf{a} \in \mathbb{R}^{N}$. Let us define, for every $\mathbf{a}^{\prime} \in \mathbb{R}^{N}$ :

$q\left(\mathbf{a}^{\prime}, \mathbf{a}\right)=\psi(\mathbf{a})+\left(\mathbf{a}-\mathbf{a}^{\prime}\right)^{\top} \nabla \psi(\mathbf{a})+\frac{1}{2}\left(\mathbf{a}-\mathbf{a}^{\prime}\right)^{\top} U(\mathbf{a})\left(\mathbf{a}-\mathbf{a}^{\prime}\right)$, where $U(\mathbf{a}) \in \mathbb{R}^{N \times N}$ is a semidefinite positive matrix. Then, $U(\mathbf{a})$ satisfies the majorant condition for $\psi$ at $\mathbf{a}$ if $q(\cdot, \mathbf{a})$ is a quadratic majorant of the function $\psi$ at $\mathbf{a}$, i.e., for every $\mathbf{a}^{\prime} \in \mathbb{R}^{N}, \psi\left(\mathbf{a}^{\prime}\right) \leq q\left(\mathbf{a}^{\prime}, \mathbf{a}\right)$.

The second optimization principle is the definition of the proximity operator of a proper, lower semicontinuous, convex function $\rho$ at $\mathbf{a} \in \mathbb{R}^{N}$, relative to the metric induced by a symmetric positive definite matrix $U$, and denoted by $\operatorname{prox}_{U, \rho}(\mathbf{a})$, which is the unique minimizer of $\rho+\frac{1}{2}(\cdot-\mathbf{a})^{\top} U(\cdot-\mathbf{a})$. When $U$ is an identity matrix of $\mathbb{R}^{N \times N}$, then this operator reduces to the original definition of the proximity operator in [16]: In this

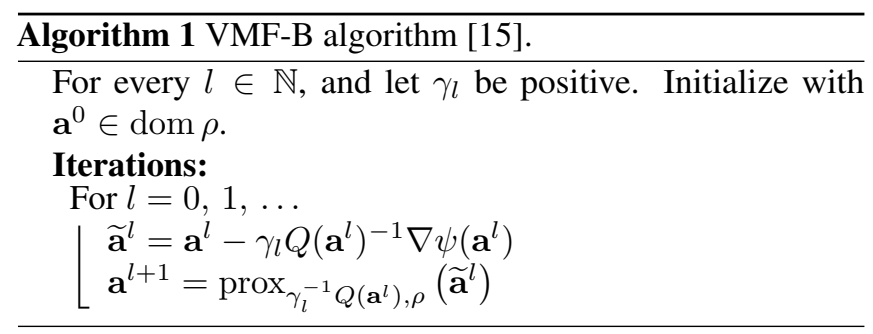

algorithm, $Q$ is a semidefinite positive matrix for building majorizing approximations of $\psi$, which is given by the following proposition established in [1]:

Proposition 1 For every $\mathbf{a} \in \mathbb{R}^{N}$, let

$$
\begin{aligned}
& \text { 1. } Q^{(1)}(\mathbf{a})=\operatorname{Diag}\left(\left(\left(a_{n}^{2}+\alpha^{2}\right)^{-1 / 2}\right)_{1 \leq n \leq N}\right), \\
& \text { 2. } Q^{(2)}(\mathbf{a})=\frac{9}{8 \eta^{2}} \mathrm{I}_{N}+\frac{1}{\ell_{1, \alpha}(\mathbf{a})+\beta} Q^{(1)}(\mathbf{a}) .
\end{aligned}
$$

where $\mathrm{I}_{N}$ is the identity matrix of $\mathbb{R}^{N \times N}$. Then, for $j \in$ $\{1,2\}, Q(\mathbf{a})=\|\mathbf{H}\|^{2} \mathrm{I}_{N}+\lambda Q^{(j)}(\mathbf{a})$ satisfies the majoration condition for $\psi$ at $\mathbf{a}$, and ${ }^{(j)}$ corresponds to two choices for $\varphi$ in Section 3.1.

To conclude, we have proposed a deconvolution method to apply to the BF-MS that imposes sparsity and regularity on the acoustic moving-source positions. This method is validated in the next section, and compared to the classical DAMAS-MS, used in acoustics for moving-source deconvolution.

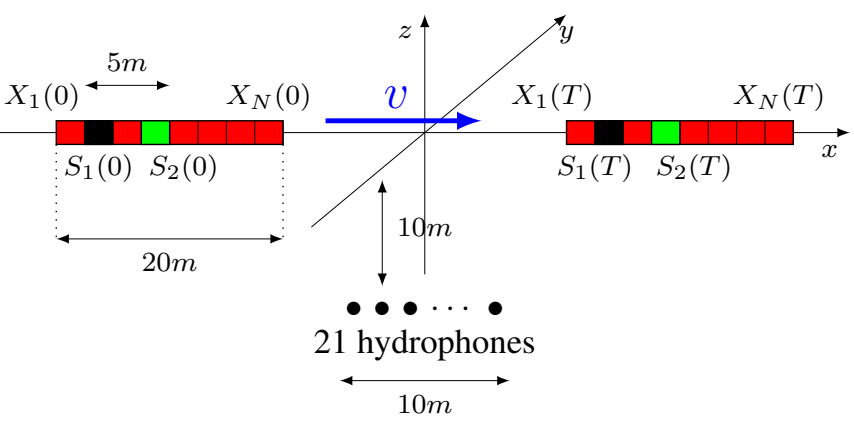

Fig. 2. Simulated configuration of a pass-by experiment. Source $S_{1}$ in black, Source $S_{2}$ in green, calculation grid in red. Blue arrow represents the global movement of the sources.

\section{SIMULATION}

The simulated configuration is presented in Fig. 2. Here, we consider two sources: a random broadband source located at $S_{1}=(-4 m, 0 m, 0 m)$ (in black) and a sum of 3 sine functions at frequencies $1200 \mathrm{~Hz}, 1400 \mathrm{~Hz}$ and $1800 \mathrm{~Hz}$ located at $S_{2}=(1 \mathrm{~m}, 0 \mathrm{~m}, 0 \mathrm{~m})$ (in green). The sources are moving jointly, following a linear trajectory of length $20 \mathrm{~m}$ at constant speed $v=2 \mathrm{~m} / \mathrm{s}$. A linear antenna of 21 hydrophones equally spaced by $0.5 \mathrm{~m}$ records the propagated acoustic signals during $T=10 \mathrm{~s}$. Zero-mean white Gaussian noise is added into the recorded signals. To perform BF-MS, the moving calculation grid $X_{n}(t), \forall n \in\{1, \ldots, N\}$ has a length of $20 m$ and contains $N=101$ points. The synthetic data were simulated using the closed form solution for the propagation of moving point sources in the free field (with a monopole radiation). For the ideal case of the simulation, the source positions matched with the positions of the grid points.

\begin{tabular}{|c|c|c|c|}
\cline { 2 - 4 } \multicolumn{1}{c|}{} & $10 \mathrm{~dB}$ & $0 \mathrm{~dB}$ & $-10 \mathrm{~dB}$ \\
\hline DAMAS-MS & 2.83 & 3.12 & 5.19 \\
\hline \hline$\ell_{1, \alpha}(\mathbf{a})$ & 1.57 & 1.58 & 1.78 \\
\hline $\log \left(\frac{\ell_{1, \alpha}(\mathbf{a})+\beta}{\ell_{2, \eta}(\mathbf{a})}\right)$ & $\mathbf{0 . 2 8}$ & $\mathbf{0 . 2 8}$ & $\mathbf{0 . 3 8}$ \\
\hline
\end{tabular}

Table 1. Results obtained by DAMAS-MS and the ones by VMF-B algorithm for the two penalty functions $\ell_{1, \alpha}$ and smoothed $\ell_{1} / \ell_{2}$, for three different SNRs. Results averaged over 200 noise realizations.

The VMF-B algorithm with the two penalty functions $\ell_{1, \alpha}$ and smoothed $\ell_{1} / \ell_{2}$ and the classical DAMAS-MS are applied to the BF-MS result. For VMF-B, the algorithm is launched on $10^{4}$ iterations and can stop earlier at iteration $l$ if $\left\|\mathbf{a}^{l}-\mathbf{a}^{l-1}\right\| \leq$ $\sqrt{N} \times 10^{-6}$.

Table 1 summarizes quantitatively the results in terms of reconstruction error on vector $\overline{\mathbf{a}}$ for different SNRs. The error is defined as the $\ell_{1, \alpha}$ norm of the error between the real $\overline{\mathbf{a}}$ and estimated $\hat{\mathbf{a}}$, which represents the ability of the method to reconstruct accurate sparse source positions. The results show that 
in all of the cases, the VMF-B algorithm with the smoothed $\ell_{1} / \ell_{2}$ has the smallest error. Consequently, in the following, we only perform the VMF-B method with the smoothed $\ell_{1} / \ell_{2}$ penalty function.

After this quantitative study, it is necessary to study the method performances qualitatively, directly on the localization maps for a SNR of $10 \mathrm{~dB}$. Fig. 3 shows the results for DAMASMS and VMF-B for two frequencies, $1200 \mathrm{~Hz}$ (a) and $770 \mathrm{~Hz}$ (b). The blue lines represent the reference sources to estimate (in terms of position and amplitude). The magenta lines are the BF-MS results, which are the starting points of the DAMASMS and VMF-B methods. The results obtained by the VMF-B algorithm are in green, and those of DAMAS-MS in red.

At the frequency $1200 \mathrm{~Hz}$ (Fig. 33) for which both sources exist, both the DAMAS-MS and VMF-B methods detect the source positions accurately. Nevertheless, DAMAS-MS gives some false alarms at $x=3 m$ and $x=5 m$, whereas VMF-B does not. These false sources have smaller amplitudes, but they are a real problem because the number of sources is generally not known.

At frequency $770 \mathrm{~Hz}$ (Fig. 33) for which only the source $S_{1}$ exists, DAMAS-MS gives a wrong result with a spatially extended source and false alarms, while the VMF-B algorithm shows an excellent result in terms of position and amplitude.

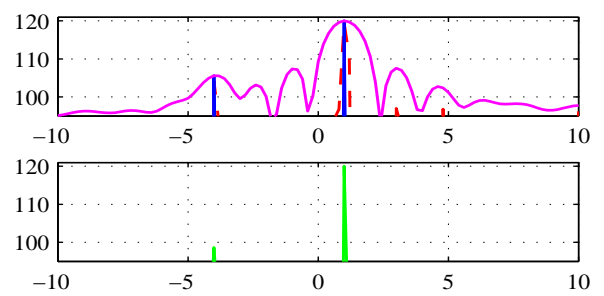

(a) At frequency $1200 \mathrm{~Hz}$

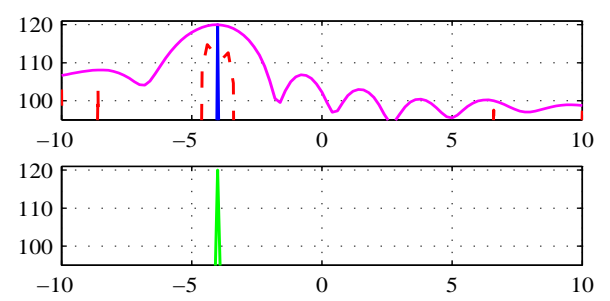

(b) At frequency $770 \mathrm{~Hz}$

Fig. 3. Autospectrum of the original sources in blue, BF-MS output in magenta, results obtained by DAMAS-MS [2] in red (top), and by VMF-B in green (bottom) for input data with $10 \mathrm{~dB}$ noise. (a) Frequency of $1200 \mathrm{~Hz}$. (b) Frequency of $770 \mathrm{~Hz}$.

The two-dimensional localization map is shown in Fig. 4 which was obtained by applying the DAMAS-MS method and VMF-B algorithm for all frequencies, where (a) is the BF-MS map, and (b) and (c) are the DAMAS-MS and VMF-B results, respectively. The first observation is that both methods localize the two sources and allow their identification as one

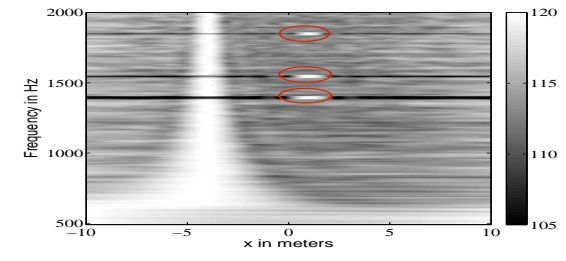

(a) Initial BF-MS

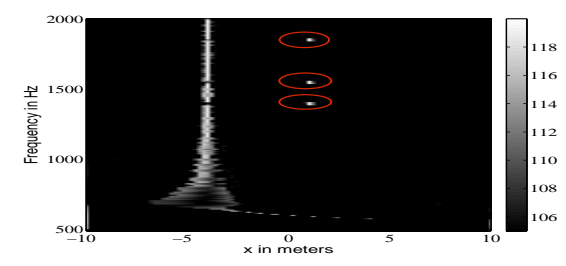

(b) Deconvolution with DAMAS-MS

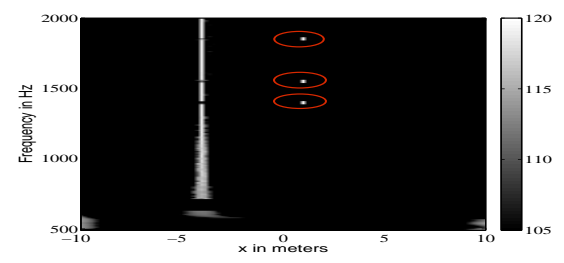

(c) Deconvolution with VMF-B

Fig. 4. Localization in the frequency-distance domain obtained. (a) Initial BF-MS (b) DAMAS-MS (c) VMF-B.

broadboand source and a sum-of-sine source.

Nevertheless, by studying the different zones indicated in red in Fig. 4, which are related to the autospectrum of the sine source at the three frequencies $1200 \mathrm{~Hz}, 1400 \mathrm{~Hz}$, and $1800 \mathrm{~Hz}$, some other conclusions can be made. The VMF-B algorithm has obviously the better performances than DAMAS-MS in term of localization, as the source $S_{2}$ is spread over several $x$ positions by DAMAS-MS, whereas VMF-B manages to estimate a point source at the true source position.

Moreover, concerning the broadband source $S_{1}$, the VMF-B algorithm has obviously better performances than DAMAS-MS, especially between the frequencies of $700 \mathrm{~Hz}$ and $1000 \mathrm{~Hz}$, where DAMAS-MS fails to localize the source.

\section{CONCLUSIONS}

This paper proposes to adapt the VMF-B algorithm [15] for moving-source localization in underwater acoustic data. As the number of sources is small enough, its autospectrum has a sparse representation, and it is possible to obtain more accurate results of deconvolution through a regularization function. In this paper, the smooth approximation of $\ell_{1} / \ell_{2}$ has shown very good performance in term of localization and suppression of false alarms. In future studies, it will be interested to validate the method with real data and to extend it to the $2 \mathrm{D}$ version, which would directly work with the entire frequency-distance domain obtained after BF-MS at all the frequencies. 


\section{REFERENCES}

[1] A. Repetti, M.-Q. Pham, L. Duval, E. Chouzenoux, and J.-C. Pesquet, "Euclid in a Taxicab: Sparse Blind Deconvolution with Smoothed $\ell_{1} / \ell_{2}$ Regularization", IEEE Signal Processing Letters, vol. 22, no. 5, pp. 539-543, Octobre 13, 2014.

[2] V. Fleury, J. Bulté, "Extension of deconvolution algorithms for the mapping of moving acoustic sources", JASA., vol. 129, no. 3, pp. 1417-1428, March 2011.

[3] R. J. Urick, " Principles of Underwater Sound”, 3rd edition, Mc Graw-Hill Book Company, 1983.

[4] J. A. Högbom, "Aperture Synthesis with a Non-Regular Distribution of Interferometer Baselines", Astron. Astrophys. Suppl., no. 15, pp. 417-426, June, 1974.

[5] P. Sijtsma, "CLEAN based on spatial source coherence", International Journal of Aeroacoustics, vol. 6, no. 4, pp. 357-374, December 17, 2007.

[6] T. F. Brooks, and W. M. Humphreys, "A deconvolution approach for the mapping of acoustic sources (DAMAS) determined from phased microphone arrays", Sound and Vibration, vol. 294, pp. 856-879, Jully 01, 2006.

[7] T. Yardibi, J. Li, P. Stoica, L. Cattafesta, "Sparsity constrained deconvolution approaches for acoustic source mapping", JASA, vol. 123, no. 5, pp. 2631-2642, June 2008.

[8] V. Fleury, J. Bulté, and R. Davy, "Determination of acoustic directivity from microphone array measurements using correlated monopoles", 29th AIAA, May 5-7, 2008, Vancouver, British Columbia Canada.

[9] T. Suzuki, “ $L_{1}$ generalized inverse beam-forming algorithm resolving coherent/incoherent, distributed and multipole sources", Journal of Sound and Vibration, vol. 330, no. 24, pp. 5835-5851, November 21, 2011.

[10] K. Sun, Y. Liu, H. Meng, and X. Wang, "Adaptive Sparse Representation for Source Localization with Gain/Phase Errors", Sensors, vol. 11, no. 5, pp. 4780-4793, May 02, 2011.

[11] J. Chen, X. Huo, "Sparse Representations for Multiple Measurement Vectors (MMV) in an Over-Complete Dictionary", ICASSP 2005, Philadelphia, PA, USA., pp. 257-260, March 2005.

[12] S. Cotter, B. Rao, K. Engan, K. Kreutz, “ Sparse solutions to linear inverse problems with multiple measurement vectors", IEEE Trans. Signal Process, vol. 53, no. 7, pp. 2477-2488, Jully 2005.
[13] N. Chu, J. Picheral, A. Mohammad-Djafari, and N. Gac, "A robust super-resolution approach with sparsity constraint in acoustic imaging", Applied Acoustics, vol. 76, pp. 197-208, Feb. 2014.

[14] B. Oudompheng, B. Nicolas and L. Lamotte, "Passive synthetic aperture array to improve noise mapping of a moving ship", OCEANS 2015, Genova, Italy, pp. 1-6, May 18-21, 2015.

[15] E. Chouzenoux, J.-C. Pesquet, and A. Repetti, "Variable metric forward-backward algorithm for minimizing the sum of a differentiable function and a convex function", J. Optim. Theory Appl., vol. 162, no. 1, pp. 107-132, Jul. 2014.

[16] P. L. Combettes and J.-C. Pesquet, "Proximal splitting methods in signal processing", in Fixed-point algorithms for inverse problems in science and engineering, $\mathrm{H}$. $\mathrm{H}$. Bauschke, R. Burachik, P. L. Combettes, V. Elser, D. R. Luke, and H. Wolkowicz, Eds., pp. 185-212. Springer Verlag, 2011.

[17] S. G. Mallat and Z. Zhang, "Matching Pursuits with Time-Frequency Dictionaries”, IEEE Trans. Signal Process, vol. 41, no. 12, pp. 3397-3415, December 1993.

[18] A. Cichocki, R. Zdunek, A. H. Phan, and S. Amari, “ Nonnegative Matrix and Tensor Factorizations: Applications to Exploratory Multi-way Data Analysis and Blind Source Separation”, Wiley Publishing, 2009. 